\title{
Structuring, Design and Analysis of a Pentab and SIW Cavity Backed Antenna for Iot Applications
}

\author{
S.P. Cowsigan ${ }^{1 *}$, D. Saraswady ${ }^{2}$ \\ ${ }^{1}$ Electronics and Communication Engineering, KPR Institute of Engineering and Technology, Coimbatore, India. \\ ${ }^{2}$ Electronics and Communication Engineering, Pondicherry Engineering College, Puducherry, India. \\ E-mail:saraswady.d@pec.edu \\ *Corresponding author E-mail:cowsigan.sp@ kpriet.ac.in
}

\begin{abstract}
Substrate Integrated Waveguide (SIW) cavity backed antenna technology is a new form of transmission line facilitating the realization of non-planar (waveguide based) circuits into planar form for easy integration with other planar (Microstrip) circuits and systems. They retain the low copper and dielectric loss property of traditional metallic waveguides and are widely used in integration of walls, floors and flame redundant wearable. SIW-CB antenna is a perfect candidate for IoT based wearable antenna with FR4 substrate. In this sense we structurizean efficient small size antenna for IoT applications to operate in the range of $5-15 \mathrm{GHz}$. FR4-epoxy substrate is chosen so that the losses are minimized hence improving the efficiency. The proposed antenna resonates at 5.4, 6.9,9.1,11.5 \& $14.2 \mathrm{GHz}$ hence forming the Pentaband with a maximum return loss of $38.6 \mathrm{db}$. The other antenna parameter values are Gain $28.5 \mathrm{db}$, efficiency $90 \%$ and VSWR 1.
\end{abstract}

Keywords: Substrate Integrated Waveguide (SIW) cavity backed, Internet of Things (IoT), Flame Redundant type 4 (FR4)

\section{Introduction}

Internet of Things (IoT) is one the trending topics which solves the demand in the Communication system. The next generations Wireless Communications are currently converging toward IoT. The IoT systems are characterized by low transmission rate and less data rate which reduces the bandwidth requirement. Owing to the connectivity characteristics of the IoT for anything at anytime and from anywhere, it is being used extensively in a wide range of industrial applications. The far-reaching integration of everyday objects into the network is a key feature of this evolution [1]. This radiating communication systems are invisibly integrated in common objects which support high data rate to meet the trending application requirements. The integration of these IoT based compact system in various objects and the antenna designs to meet the ever increasing demand for data rate are the major challenges. As interest in the commercialization of IoT has increased over the past few years, Antenna designers have started developing different antenna topology that ensures stability, high gain, better efficiency and resonating property. The other challenges are designing an antenna which has low profile, conformable, low cost and easily mountable device. Implementing the antenna directly in the object's material leads to a significant size, shape and cost reduction, since the readily available material and dimensions of the object itself are reused for the development of antenna. Hence, novel materials should be considered as antenna substrates [2]. Furthermore, an antenna topology with high isolation between the antenna and its environment is required. This minimizes detuning due to objects in the antenna's near field and offers stable high radiation efficiency in different environments.

\section{SIW Technology and Its Benefits}

SIW-CB antenna technology is the perfect candidate for the above mentioned challenges. SIW technology exhibits very attractive features for IoT applications. Both active and passive components can be implemented on a single substrate by means of a low-cost production process, paving the way for a low-profile and costeffective realization of a entire circuit. For IoT applications, the object's materials can be reused to implement the SIW structure, provided that a dedicated material characterization technique is involved. Such an approach yields a significant cost and area reduction, as the readily available material and area of the object itself are exploited to construct the complete circuit[3].SIW technology may be combined with innovative substrate materials to obtain a green, stable and high-performance broadband design.SIW structures on textile were initially developed for the specific application in localization system for firefighters [4][5]. The wearable components, in this case must meet the requirements of fire resistance and, at the same time, they should be flexible, to allow natural movements.

The Microstrip and other antenna technologies are largely affected by the undesired radiation and surface waves. These losses are minimized by restricting the field inside the SIW structure. The arrangement of the rows of via holes at sides acts as effective electric walls which help in confining the fields within the SIW core. By having such an arrangement the SIW topology has the benefits of reduced undesired radiation, surface waves and other losses. Further SIW technology provides a high radiation efficiency and a good front-to-back ratio (FTBR) with surface wave suppression. The antenna is isolated from its environment due to the antenna cavity that contains the field, hence SIW has 
high isolation factor. This makes them excellently suited for very low-power and battery-powered applications.

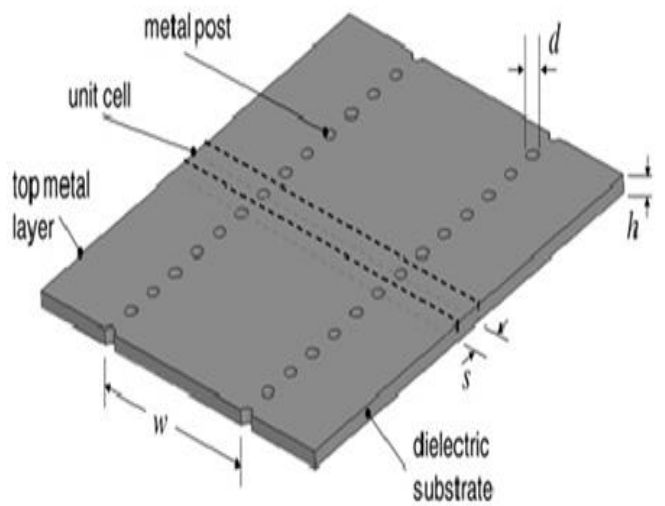

Fig. 1: SIW technology

\section{SIW Cavity Backed Antenna}

\section{A. Introduction}

SIW Cavity Backed (SIW-CB) antennas using the SIW Technology is equivalent to the classical rectangular Wave guides in displaying the propagation and dispersion characteristics including the E-field pattern. SIW-CB antenna includes most of the advantages of conventional metallic waveguides namely high quality-factor and high power-handling capability. In addition to this SIW has excellent integrating property which helps in the convenient integration of additional components like selective filters and power amplifier with the chip-set. SIW structure is fabricated by using two rows of conducting cylinders or slots embedded in a dielectric substrate that electrically connect two parallel metal plates. These metal via holes can be placed at the sides or anywhere in the antenna. In this way, the non-planar rectangular waveguide can be made in planar form, compatible with existing planar processing techniques (e.g.Printed Circuit Board (PCB) or Low-Temperature Co-fired Ceramic (LTCC) technology) [6] .

\section{B. Operation Principles}

SIW structures displays radiation characteristics similar to those of rectangular waveguides, provided that the metallic via holes are closely spaced and radiation leakage can be neglected (Fig. 2). In general, SIW modes practically coincide with a subset of the guided modes of the rectangular waveguide, namely with the $\mathrm{TE}_{\mathrm{n} 0}$ modes, with $n 1 / 41,2 \ldots$ TM modes are not supported by SIW due to the gaps between metal vias. In fact the transverse magnetic field determines the longitudinal surface currents which are subject to strong radiation due to the presence of gaps [7]. The Fundamental mode is similar to the $\mathrm{TE}_{10}$ mode of a rectangular waveguide with vertical electric current density on the side walls.[10]

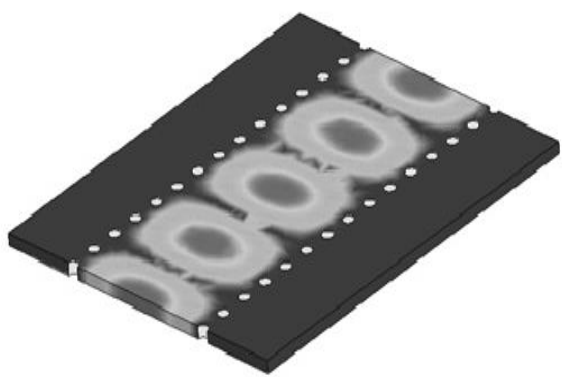

Fig. 2: SIW-CB antenna radiation mode

\section{Siw-Cb Design}

The SIW-CB antenna has two rows of metal vias structured in dielectric substrate. Resonant or cut-off frequency is independent of the substrate thickness and the dielectric loss is inversely proportional to thickness of the substrate. These relations help in determining the preliminary dimensioning and designing of SIW components without any full wave analysis tools. The most popular relation and Design formula was derived in [8]

$$
\begin{gathered}
\mathrm{W}_{\text {eff }}=\mathrm{W}+(\mathrm{d} /(0.95) \mathrm{s}) \\
\mathrm{W}_{\text {eff }}=\mathrm{W} / \sqrt{\varepsilon}
\end{gathered}
$$

Where $\mathrm{W}_{\text {eff }}$ effective width of the dielectric

W-transverse spacing between the two rows of metal vias

$\mathrm{s}$ - distance between the vias

$\mathrm{d}$ - diameter of the via hole

$\mathrm{h}-$ thickness of the dielectric substrate

$\varepsilon_{\mathrm{r}}$ - relative permittivity of substrate

\section{Proposed Antenna}

The modern technological antenna consists of few design specifications, of which the frequency of operation, feeding technique, dielectric substrate and its thickness are important. The proposed antenna has been structured and designed to operate in the lower $\mathrm{GHz}$ frequency range for IoT applications from $5-15$ $\mathrm{GHz}$ with resonance at five different frequencies. Coaxial or probe feeding technique has been employed to reduce the radiation losses. The inner conductor of the coaxial connector extends through the dielectric and is soldered to the radiating patch, while the outer conductor is connected to the ground plane. [9]

The dielectric substrate has been carefully chosen to perfectly suit the IoT environment and to fabricate in flame resistant areas for achieving efficient propagation characteristics. As FR4- epoxy substrate is answering the above mentioned requirements, it has been selected with these specification, relative permittivity of $\varepsilon_{\mathrm{r}}$ 4.4 and dielectric loss tangents of 0.02 . As we have seen in the above equations, thickness of the substrate has a major role in determining the size of the SIW-CB. The losses in the SIW structure are also need to be considered in finalizing the substrate thickness, thicker substrate reduces conductor losses whereas increasing the dielectric losses, hence thickness has to be optimized. The measured thickness of the substrate is $0.508 \mathrm{~mm}$. The proposed SIW-CB antenna has overall dimension of $26 * 25 * 0.508 \mathrm{~mm}^{3}$ as shown in Fig. 3 .

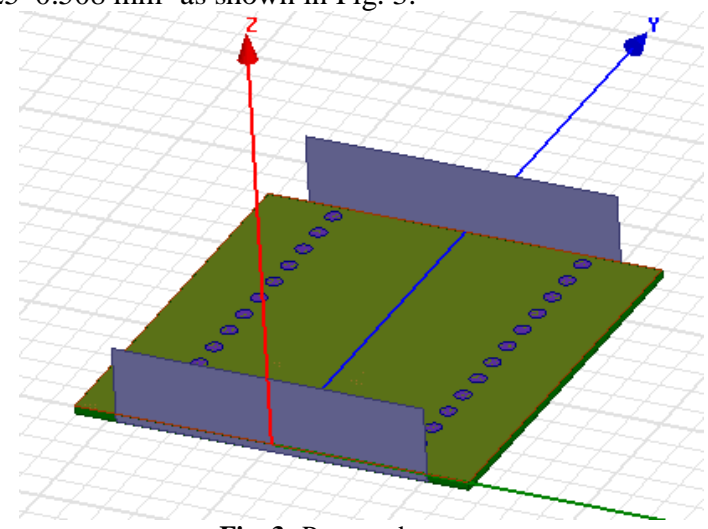

Fig. 3: Proposed antenna

\section{Results and Discussions}

The proposed SIW-CB antenna has the advantage of resonating at five different frequencies in the range $5-15 \mathrm{GHz}$ for IoT applications. The five resonant frequencies are $5.4 \mathrm{GHz}, 6.9 \mathrm{GHz}$, $9.1 \mathrm{GHz}, 11.5 \mathrm{GHz} \& 14.2 \mathrm{GHz}$ with a maximum return loss of $36.5 \mathrm{db}$ occurring at $14.2 \mathrm{GHz}$. The return losses for the other frequencies are $19.5 \mathrm{db}, 28 \mathrm{db}, 32 \mathrm{db} 33.5 \mathrm{db}$ respectively as shown in Fig. 4. The above mentioned frequencies help in the 
direct operation of various devices components equipments and electronic gadgets through internet. The advantage of this SIW$\mathrm{CB}$ structure is that it works in five various frequencies. These electronic appliances can be controlled using the proposed antenna by fabricating it in any of the flame redundant materials.

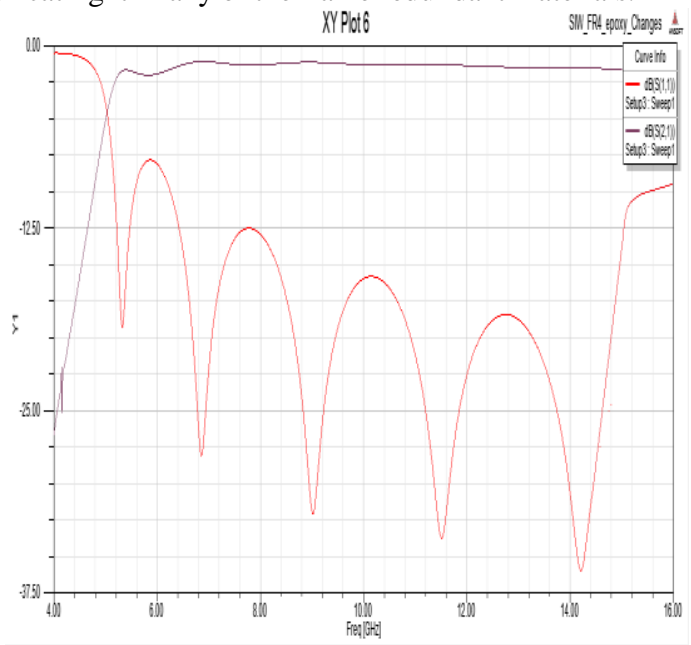

Fig. 4: Return loss characteristics

The VSWR measures almost 1 over the entire range of operation 5 - $15 \mathrm{GHz}$ which is an excellent value to achieve. In the range from 5.7 to $6.2 \mathrm{GHz}$, the VSWR value is 2 which is also in the acceptable range. The overall VSWR characteristic as shown in Fig. 5 has revealed that the Standing wave losses have been reduced considerably. The Simulated results are obtained using the An soft simulation software High Frequency Structure Simulator (HFSS).

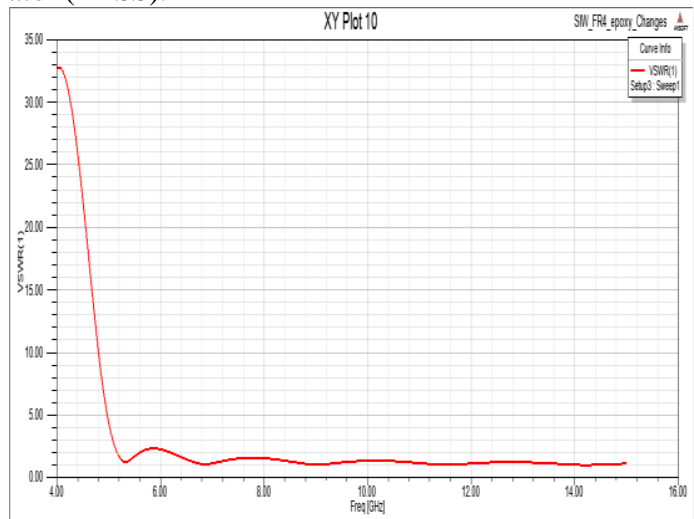

Fig. 5: VSWR

The proposed SIW-CB antenna covers the frequency range for IoT systems between $5-15 \mathrm{GHz}$ with a Pentaband characteristics and the radiation pattern including both far field and near field patterns are directional.

\section{Conclusion}

In this paper, a compact SIW technology implemented cavity backed antenna is proposed for IoT applications to control various electronic appliances. The antenna has simple configuration and is easy to fabricate in flame redundant materials. The benefits of SIW technology have been combined with FR4 substrate to produce Pentaband frequency. The SIW-CB antenna has been structured designed and analyzed to covers the frequency range between $5-15 \mathrm{GHz}$ to generate pentaband characteristics. Simulated return loss and VSWR results show that the proposed antenna is a good candidate for IoT applications.

\section{References}

[1] Sund-maeker H, Guillemin P, Friess P \& Woelfflé S, "Vision and Challenges for Realising the Internet of Things", Luxembourg. Publications Office of the European Union, (2010).

[2] Olivier C, Sam L, Sam A, Dries VG, "Half-Mode Substrate Integrated Waveguide Cavity Backed Slot Antenna on Cork Substrate", IEEE Transaction Antennas and Propagation, Vol.49, (2001), pp.1020-1024.

[3] Bozzi M \& Moro R, "Low-cost fabrication, eco-friendly materials, and easy integration: the new technological paradigm for the future wireless sensor networks", European Microwave Conference (EuMC), Nuremberg, Germany, (2013), pp.858-861.

[4] Moro R, Agneessens S, Rogier H \& Bozzi M, "Wearable Textile Antenna in Substrate Integrated Waveguide Technology", IET Electronics Letters, Vol.48, No.16, (2012), pp.985-987.

[5] Moro R, Agneessens S, Rogier H, Dierck A \& Bozzi M, "Textile Microwave Components in Substrate Integrated Waveguide Technology", IEEE Transactions on Microwave Theory and Techniques, Vol.63, No.2, (2015), pp.422-432.

[6] Bozzi M, Georgiadis A \& Wu K, "Review of substrateintegrated waveguide circuits and antennas", IET Microwave Antennas Propagation, Vol.5, No.8, (2011), pp.909-920.

[7] Daniels RC \& Heath RW, "60 GHz Wireless Communications: emerging requirements and design recommendations", IEEE Veh. Technology Mag., Vol.2, No.3, (2007), pp.41-50.

[8] Cassivi Y, Perregrini L, Arcioni P, Bressan M, Wu K \& Conciauro $\mathrm{G}$, "Dispersion characteristics of substrate integrated rectangular waveguide", IEEE Microwave Wireless Components Letter, Vol.12, No.9, (2002), pp.333-335.

[9] G, Abikhanova, A Ahmetbekova, E Bayat, A Donbaeva, G Burkitbay (2018). International motifs and plots in the Kazakh epics in China (on the materials of the Kazakh epics in China), Opción, Año 33, No. 85. 20-43.

[10] G Ainabekova, Z Bayanbayeva, B Joldasbekova, A Zhaksylykov (2018). The author in esthetic activity and the functional text (on the basis of V. Mikhaylov's narrative ("The chronicle of the great jute"). Opción, Año 33. 63-80. 